\title{
液态泡沫强制渗流波叠加现象及其能量分析
}

\author{
孙其诚 $^{(1)}$, 黄晋 ${ }^{(2)}$, 王光谦 ${ }^{(1}$ \\ (1) 清华大学水沙科学与水利水电工程国家重点实验室, 北京 100084; \\ (2) Institute for Corrosion and Multiphase Technology, Ohio University, Ohio 45701, USA \\ E-mail: qcsun@tsinghua.edu.cn
}

2008-03-20 收稿, 2008-06-05 接受

国家自然科学基金(批准号: 20776142)和人事部留学回国人员择优项目(2007 年)资助

摘要 液态泡沫是大量气泡密集堆积在微量表面活性剂溶液中形成的复杂体系, 探讨其稳 定性是近 20 年来泡沫物理研究的重点, 其中渗流(亦即内部微量溶液的流动)对泡沫稳定性 的影响已得到深入研究. 基于前期泡沫结构、渗流力学和能量分析等一系列工作, 数值模拟 了一维多重渗流波的传播和重叠现象, 发现了波速的线性叠加规律, 这是液态泡沫强制渗 流所特有的; 理论分析了渗流波重叠前后㮀性耗散和表面能的变化.

关键词

液态泡沫

渗流

稳定性条件

软凝聚态物质 液态泡沫(liquid foams)是大量气泡密集堆积在 少量表面活性剂溶液中形成的整体, 分为液膜、柏拉 图通道和结点三种基本结构 ${ }^{[1,2]}$. 泡沫中的微量液体 在重力(或外界压差力)、毛细管力以及黏滞力共同作 用下, 在柏拉图通道及结点形成的网络内发生流动, 称为泡沫渗流. 在几种渗流方式中, 强制渗流是指以 恒定流率输入与泡沫中溶液成分一致的液体而引起 的泡沫渗流，其特征现象是形成一个以恒定速率传 播的渗流波, 如图 1(a)所示. 强制渗流易于理论研究 和实验观测, 通过测定该渗流波传播速率以及液体 体积分数的演变规律, 就可以推断内部柏拉图通道 的界面性质、微流动形态及黏性能量耗散等信息, 进 而分析其对泡沫稳定性的影响, 这是近 20 年来泡沫 稳定性机理研究和工艺控制的重点之一 ${ }^{[3]}$.

除了在泡沫结构理论方面的工作外 ${ }^{[4 \sim 6]}$, 我们还 进行了一系列泡沫强制渗流的实验测量、理论分析和 数值模拟 ${ }^{[7]}$, 得到了一维液态泡沫强制渗流中渗流波 传播规律以及液体体积分数的时空演变规律. 研究发 现，在我们的实验中泡沫柏拉图通道上的微流动为泊 肃叶流, 进而推断能量耗散主要发生在柏拉图通道内, 得到了泡沫基本气泡单元(采用Kelvin cell)的綮性耗散 能表达式, 并依据Surface Evolver软件 ${ }^{[8]}$ 计算了不同液 体体积分数时Kelvin气泡单元的表面能. 同时, 我们

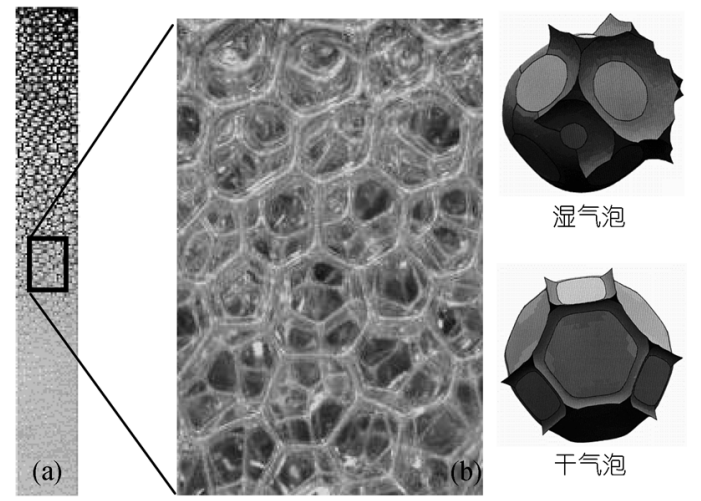

图 1 一维泡沫强制渗流实验

(a) 微量液体以恒定流率从顶端输入, 在重力作用下产生强制渗 流波向下传播; (b) 渗流波干湿泡沫分界面处的细观泡沫结构及 Kelvin 气泡单元(Kelvin cell)

基于柏拉图通道内微量液体微元所受重力(或外界压 差力)、毛细管力以及秥滞力的平衡, 建立了两维渗流 方程, 研究了重力对两维液态泡沫强制渗流的影响 ${ }^{[9]}$. 总体来说, 单输入渗流波的传播规律比较透彻 ${ }^{[10]}$.

在实际液态泡沫中, 内部液体或外部输入液体 的空间分布通常不均匀, 内部气泡粗化(coarsing)或 破裂(film rupture)也使得局部液体富集, 这样泡沫内 部多种渗流方式会同时或相继发生、相互影响, 对泡 沫结构和稳定性造成不可预测的干扰，这必然是复 
杂的非线性力学问题, 其规律目前尚不清晰. 一个典 型应用例子就是铝及铝合金熔体泡沫制备过程中的 泡沫渗流规律以及泡沫稳定性控制问题 [11,12]. 我们 曾对两维泡沫中双点平行输入时强制渗流波的相互 作用对传播速率的影响进行了实验测定和数值计算, 发现了一些新现象 ${ }^{[13]}$, 但是多个渗流波间耦合、叠 加现象及内在机理分析仍然不全面和深入.

本文在现有工作基础上，对多股强制渗流波在 一维泡沫中传递和叠加现象进行了数值模拟, 量化 了重叠前后波速之间的关系，并对该过程中表面能 和黏性耗散变化进行了分析.

\section{1 强制渗流方程}

当液态泡沫内发生渗流时, Hutzler等人 ${ }^{[14]}$ 和孙 其诚等人 ${ }^{[9]}$ 假设柏拉图通道是直的, 并随着液体体 积分数的大小而扩展或收缩; 同时流体在柏拉图通 道边壁上无滑移, 亦即泊肃叶流, 则两维渗流方程为

$$
150 \mu \frac{\partial A}{\partial t}+\rho g \frac{\partial A^{2}}{\partial y}-\frac{1}{3} C \gamma\left[\frac{\partial^{2}}{\partial y^{2}}\left(A^{3 / 2}\right)+\frac{\partial^{2}}{\partial x^{2}}\left(A^{3 / 2}\right)\right]=0,
$$

式中各项分别为黍滞力、重力和毛细管力, 其中 $A$ 为 柏拉图通道横截面积 $(A \sim \phi, \phi$ 为液体体积分数 $), C$ 为 柏拉图通道截面结构常数, $C=\left(3^{1 / 2}-\pi / 2\right)^{1 / 2}, \gamma$ 和 $\mu$ 分别 为液体的黏滞系数和表面张力系数, $t$ 为时间, $x$ 和 $y$ 分别为水平方向和重力方向位置. (1)式的无量纲形 式为

$$
\frac{\partial \phi}{\partial \tau}+\frac{\partial}{\partial \xi}\left(B_{0} \phi^{2}-\frac{\sqrt{\phi}}{2} \frac{\partial \phi}{\partial \xi}\right)-\frac{\partial}{\partial \eta}\left(\frac{\sqrt{\phi}}{2} \frac{\partial \phi}{\partial \eta}\right)=0 .
$$

其中 $\xi=y / x_{0}, \eta=x / x_{0}, \tau=t / t_{0}, A=\phi x_{0}{ }^{2} . x_{0}$ 为柏拉图通 道的长度, $x_{0}=2^{7 / 12} 12^{-1 / 2} V_{\mathrm{b}}{ }^{1 / 3}$ ( $V_{\mathrm{b}}$ 为气泡的体积); 时间 尺度 $t_{0}=150 \mu x_{0} /(C \gamma)$; 无量纲因子(Bond number) $B_{0}=$ $\rho g x_{0}{ }^{2} /(\mathrm{C} \gamma)$ 表示重力和表面张力对渗流的相对影响, $g=9.8 \mathrm{~m} / \mathrm{s}^{2}$.

图 2 是模拟采用的 Hele-Shaw cell 长管, 高为 20 $\mathrm{cm}$ 、横截面 $(w \times D)$ 为 $1 \mathrm{~cm} \times 1 \mathrm{~cm}, w$ 为水平方向输入 宽度, $D$ 为 Hele-Shaw cell 前后面间的厚度, 管内充满 直径为 $1.1 \mathrm{~mm}$ 气泡组成的泡沫. 在泡沫顶部横截面 以恒定流率输入表面活性剂溶液, 其物性取水在 $20^{\circ} \mathrm{C}$ 的物性, $\mu=1 \times 10^{-3} \mathrm{~kg} /(\mathrm{s} \cdot \mathrm{m}), \rho=1 \times 10^{3} \mathrm{~kg} / \mathrm{m}^{3}$, 表面 张力系数 $\gamma=2.5 \times 10^{-2} \mathrm{~kg} / \mathrm{s}^{2}$ 是水表面张力的 $1 / 3$, 则 $t_{0}=5.75 \times 10^{-3} . x_{0}=3.84 \times 10^{-4} \mathrm{~m}, B_{0}=0.144$. 实验中微量 液体输入流率 $Q_{\exp }$ 与数值模拟流率 $Q_{\mathrm{sim}}$ 的关系为
$Q_{\text {sim }}=Q_{\text {exp }} t_{0} /\left(x_{0}{ }^{2} D\right)$, 而 $Q_{\text {sim }}=\phi_{0}^{2} B_{0} w / \delta \eta$, 其中 $\delta \eta$ 为数 值计算时水平输入宽度方向的网格步长, 在本文中 $w / \delta \eta=26$, 则有 $\phi_{0}=1.023 Q_{\exp }{ }^{1 / 2}$ (其中 $Q_{\exp }$ 的单位为 $\mathrm{mL} / \mathrm{s}$ ), 这样在求解 (2)式时作为边界条件直接在顶部 横截面上给定 $\phi_{0}$, 而初始时泡沫内部干燥, 各处 $\phi=$ $0.005 \%$. 边界条件为

$$
\left.\frac{\partial \phi}{\partial \eta}\right|_{\eta=0}=0,\left.\frac{\partial \phi}{\partial \eta}\right|_{\eta=w / x_{0}}=0,\left.\quad \phi\right|_{\xi=0}=\phi_{0} .
$$

本文中模拟了 $t=170 \mathrm{~s}$ 时的强制渗流, $t \leqslant 56 \mathrm{~s}$ 时, 初始输入流率为 $Q_{\text {exp }}=1.52 \times 10^{-3} \mathrm{~mL} / \mathrm{s}$, 对应顶部横截 面上的 $\phi_{0}=4 \%$; 之后输入流率相继增加两次: (i) $t>56$ $\mathrm{s}$ 时, $Q_{\text {exp }}=3.44 \times 10^{-3} \mathrm{~mL} / \mathrm{s}, \phi_{0}=6 \%$, 可以观测到一个 渗流波合并现象, 见图 2(b); (ii) $t>92 \mathrm{~s}, Q_{\exp }=$ $4.68 \times 10^{-3} \mathrm{~mL} / \mathrm{s}, \phi_{0}=7 \%$, 可以产生两次渗流波合并, 进而对渗流波传播规律予以量化.

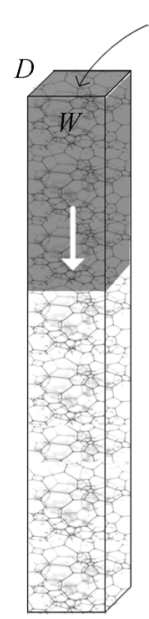

(a)

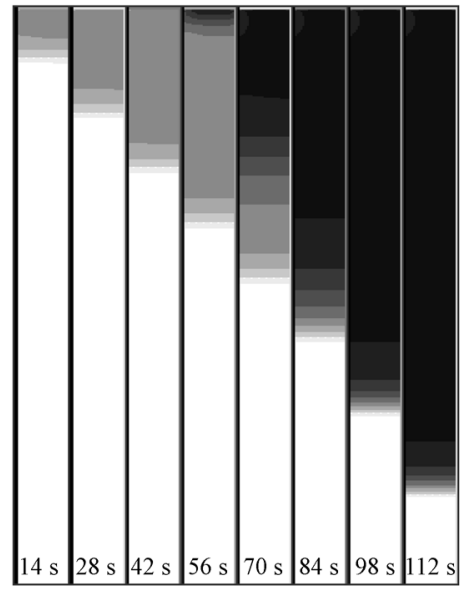

(b)
图 2 一维强制渗流示意图

（a）计算中采用的充满干泡沫的 Hele-Shaw cell; (b) 双强制渗流 波的传播和合并过程. 首先输入小流率溶液, $56 \mathrm{~s}$ 后加大流率, 这 样各产生一个渗流波, 而后者速度更高, 最终赶上第一个渗流波 而合并为一个波向下传播

\section{2 结果与讨论}

\section{1 强制渗流波速叠加}

本文采用中心差分法求解(2)式, 结果见图 2 和 3 . $t<56 \mathrm{~s}$ 时在泡沫顶部以较小流率输入液体 $\left(Q_{\exp }=\right.$ $\left.1.52 \times 10^{-3} \mathrm{~mL} / \mathrm{s}\right)$, 使得泡沫液体体积分数稍微增加, 在泡沫内形成一个渗流波(见图 2(b)和图 3中 $A$ 区) 以 速度 $v_{a}$ 向下传播; $t>56 \mathrm{~s}$ 时增加输入流率 $\left(Q_{\exp }\right.$ $\left.=3.44 \times 10^{-3} \mathrm{~mL} / \mathrm{s}\right)$, 在湿泡沫基础上产生第二个渗流 


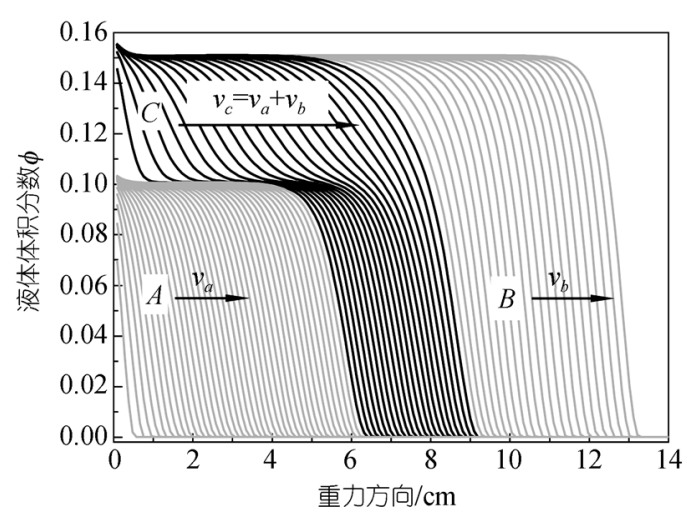

图 3 双强制渗流引起的液体体积分数沿重力方向的演变 各线间的时间间隔均为 $1.43 \mathrm{~s}, A$ 区和 $C$ 区相继发生强制渗流波, $B$ 区是两波合并后形成的新渗流波

孤立波以速度 $v_{c}$ 传播, 显然 $v_{c}>v_{a}$ (见图 2(b)和图 3 中 $C$ 区). 经过一段时间后，第二个孤立波会追赶上 第一个波, 合并为一个波恒速沿重力方向传播, 速度 为 $v_{b}$ (见图 2(b)和图 3 中 $B$ 区).

较小流率 $Q_{a}$ 产生的波速为 $v_{a}$, 由于是泊肃叶流 动, 则

$$
Q_{a} \sim v_{a}^{2}, v_{a} \sim \phi_{a} .
$$

当流率增加至 $Q_{b}$ 时, 两渗流波合并后的渗流波速为 $v_{b}$, 则

$$
Q_{b} \sim v_{b}^{2}, v_{b} \sim \phi_{b} .
$$

那么 $\Delta Q=Q_{b}-Q_{a}=v_{b}^{2}-v_{a}^{2}$, 相应的 $\phi$ 增量为

$$
\Delta \phi=v_{b}-v_{a} .
$$

对于第二个渗流波已经形成、以速度 $v_{c}$ 传播但尚末 与第一个波合并的区域 $C$ 内(见图 3), 有 $\Delta Q=v_{c} \Delta \phi$, 由 (4) (6)式得到

$$
v_{c}=v_{b}+v_{a} .
$$

可以推知, 当 $Q_{a}$ 和 $Q_{b}$ 相差不大时, 则有 $v_{c} \approx 2 v_{a}$.

从图 3 计算得到 $v_{A}=0.103 \mathrm{~cm} / \mathrm{s}, v_{B}=0.144 \mathrm{~cm} / \mathrm{s}$, $v_{C}=0.245 \mathrm{~cm} / \mathrm{s}$ ，则有 $v_{C} \approx v_{B}+v_{A}$. Hutzler等人 [15]曾采 用 $\mathrm{AC}$ 电容方法实验测量了双渗流波的速度叠加, 在 实验允许误差内所得结果也与理论估算符合较好.

我们还进行了多股强制渗流波叠加现象的模拟. $t<56 \mathrm{~s}$ 时 $Q_{\mathrm{exp}}=1.52 \times 10^{-3} \mathrm{~mL} / \mathrm{s}, 56 \mathrm{~s}<t<92 \mathrm{~s}$ 时 $Q_{\text {exp }}=3.44 \times 10^{-3} \mathrm{~mL} / \mathrm{s}, t>92 \mathrm{~s}$ 时 $Q_{\text {exp }}=4.68 \times 10^{-3} \mathrm{~mL} / \mathrm{s}$, 结果如图 4 所示.

图 4 中 $v_{D}=0.185 \mathrm{~cm} / \mathrm{s}, v_{B}=0.144 \mathrm{~cm} / \mathrm{s}, v_{E}=0.312$ $\mathrm{cm} / \mathrm{s}$, 则 $v_{E} \approx v_{B}+v_{D}$. 对比图 3 和图 4, 发现 $v_{A} / \phi_{A} \approx$

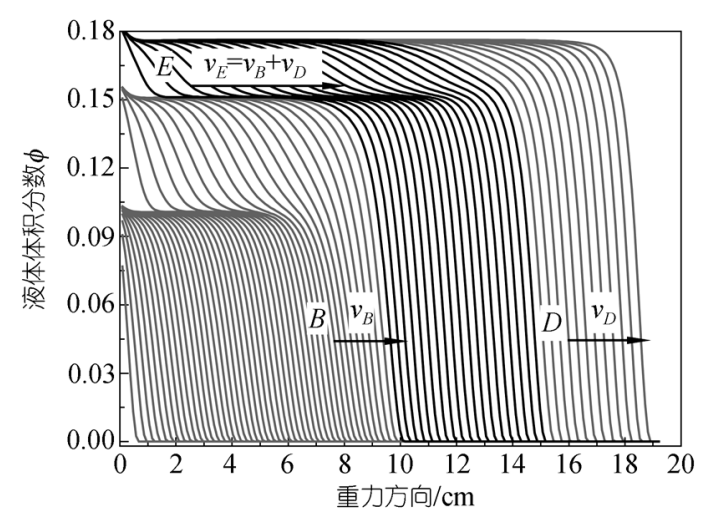

图 4 三股强制渗流引起的液体体积分数沿重力方向的演变 各线间的时间间隔均为 $1.98 \mathrm{~s} ; B$ 区是前两次渗流波合并后的单一

波, 与 $E$ 区新渗流波又发生重叠, 合并为 $D$ 区的单一波

$v_{B} / \phi_{B} \approx v_{D} / \phi_{B} \approx 0.97 \pm 0.04$, 与泊肃叶流动情况时的 $v_{a} \sim \phi_{a}$ 理论分析结果一致.

\section{2 气泡单元上黏性流动耗散和表面能: $E_{\mu}, E_{s}$}

在强制渗流中, 渗流波经过的泡沫区域内液体 体积分数基本一致, 因此分析泡沫内的流动耗散和 表面能就简化为分析泡沫内气泡单元上的流动耗散 和表面能, 而气泡单元往往简化为Kelvin单元结构 (见图 1(b)). 我们对此进行了推导 ${ }^{[7]}$, 把流体流过单 个柏拉图通道需要的时间作为Kelvin结构体内流动 的特征时间 $\tau$, 在时间 $\tau$ 内单个Kelvin气泡上流动耗散 为

$$
E_{\mu}=\rho g R^{4}\left(0.0439 \phi^{5 / 2}+0.0103 \phi^{2}\right) .
$$

我们采用软件Surface Evolver ${ }^{[8]}$ 模拟得到了不同 $\phi$ 的 Kelvin结构, 得到相应的Kelvin结构表面能为

$$
E_{s}=\gamma R^{2}\left(30.917-10.849 \phi^{1 / 2}-9.161 \phi\right) .
$$

从(8), (9)式和图 5 可以看出, 当输入流率较大时, 泡沫内 $\phi$ 也较大 $\left(\phi \sim Q_{\exp }{ }^{1 / 2}\right)$, 柏拉图通道内液体流动速 度及黏性耗散快速增加 $\left(E_{\mu} \sim \phi^{2.5}\right)$; 而 $\phi$ 的增加使得液 体压强增加, 气液界面受表面张力而收缩, 表面能缓 慢减小 $\left(E_{s} \sim \phi^{0.5}\right)$; 反之亦然. 从图 5 的计算结果中可以 看出: $A, B$ 和 $D$ 区的 $\phi$ 分别为 $0.1,0.15$ 和 0.175 , 对应 的 $E_{s}$ 从 $2.01 \times 10^{-7} \mathrm{~J}$ 减小到 $1.87 \times 10^{-7} \mathrm{~J}$, 而 $E_{\mu}$ 从 $2.5 \times 10^{-12} \mathrm{~J}$ 增加到 $4.96 \times 10^{-12} \mathrm{~J}$.

\section{3 结论}

通过对高为 $20 \mathrm{~cm}$ 、横截面为 $1 \mathrm{~cm} \times 1 \mathrm{~cm}$ 的 HeleShaw cell 长管内多股强制渗流波追赶及叠加现象的 


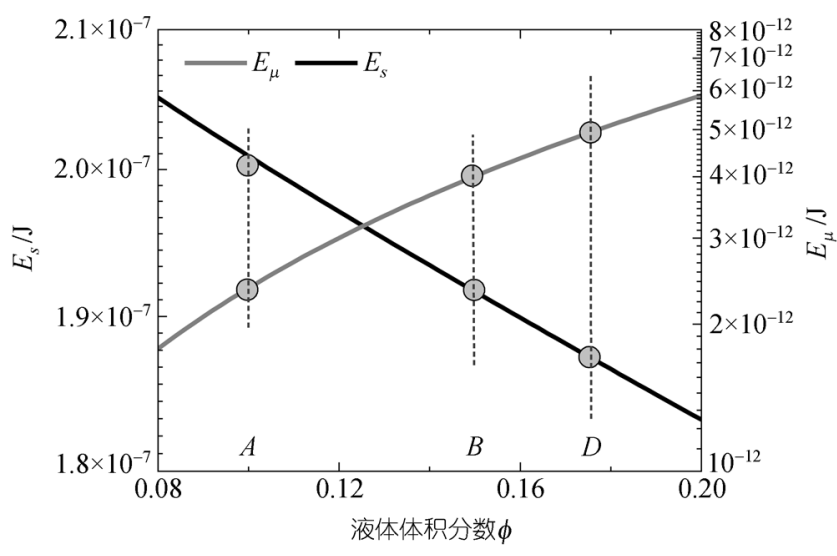

图 5 渗流波合并前后黏性耗散和表面能的变化 $A$ 区、 $B$ 区和 $D$ 区的位置见图 3 和图 4

模拟，发现了波速的线性叠加规律，与常见机械波的 叠加规律不同. 对渗流波内黍性耗散和表面能的分 析表明，渗流波重叠前后的黏性耗散变化很大，而表
面能变化微小. 本文结果可以初步应用于熔融金属 泡沫的制备工艺中, 分析多重渗流波对泡沫结构的 影响等.

\section{参考文献}

Plateau J A F. Statique Expérimentale et Théorique des Liquides Soumis aux Seules Forces Moléculaires. Paris: Gauthier Villars, 1873 孙其诚，黄晋. 液态泡沫结构及其稳定性. 物理, 2006, 35(12): 1050-1054

Weaire D, Hutzler S. The Physics of Foams. Oxford: Oxford University Press, 1999

Sun Q C, Hutzler S. Lattice gas simulations of 2D liquid foams. Rheologica Acta, 2004, 43: 567-574[DOI]

Sun Q C, Hutzler S. Studying localized bubble rearrangements in 2D liquid foams using a hybrid lattice gas model. Coll Surf A, 2005, 263(1-3): $27-32[\mathrm{DOI}]$

Sun Q C, Hutzler S. Light scattering through 2D plateau borders and foams. Coll Surf A, 2007, 309(1-3): 182-188[DOI]

Huang J, Sun Q C. Experimental study and analysis of energy evolution of liquid foam drainage in one dimension. Acta Phys Sin, 2007, 56(10): 6124-6131

Brakke K. The surface evolver. Exp Math, 1992, 1(2): 141-165

孙其诚，葛蔚，黄晋. 重力对两维液态泡沫点输入强制渗流的影响. 科学通报, 2006, 51(18): 2104一-2108

黄晋, 孙其诚. 液态泡沫渗流的机理研究进展. 力学进展, 2007, 37(2): 269-278

尚金堂, 何德坪. 铝合金熔体中球形泡沫的生长. 中国科学 B 辑: 化学, 2005, 35(3): 212-219

Banhart J, Weaire D. On the road again: Metal foams find favor. Phys Today, 2002, 55: 37-42[DOI]

Huang J, Sun Q C. Foam drainage with twin inputs in two dimensions. Coll Surf A, 2007, 309(1-3): 132-136[DOI]

Hutzler S, Cox S, Wang G. Foam drainage in two dimensions. Coll Surf A, 2005, 263: 178-183[DOI]

Hutzler S, Verbist G, Weaire D, et al. Measurement of foam density profiles using AC capacitance. Europhys Lett, 1995, 31(8): 497$502[\mathrm{DOI}]$ 